\title{
Human PBMC-transferred murine MHC class I/II-deficient NOG mice enable long-term evaluation of human immune responses
}

\author{
Tomonori Yaguchi ${ }^{1,5,6}$, Asuka Kobayashi ${ }^{1,5}$, Takashi Inozume ${ }^{2}$, Kenji Morii ${ }^{1}$, Haruna Nagumo ${ }^{1}$,
} Hiroshi Nishio ${ }^{1,3}$, Takashi Iwata ${ }^{1,3}$, Yuyo $\mathrm{Ka}^{4}$, Ikumi Katano ${ }^{4}$, Ryoji Ito ${ }^{4}$, Mamoru Ito ${ }^{4}$ and Yutaka Kawakami ${ }^{1,6}$

Immunodeficient mice engrafted with human peripheral blood cells are promising tools for in vivo analysis of human patient individual immune responses. However, when human peripheral blood mononuclear cells (PBMCs) are transferred into NOG (NOD/Shi-scid, IL-2rgnul) mice, severe graft versus host disease (GVHD) hinders long term detailed analysis. Administration of human PBMCs into newly developed murine MHC class I- and class II-deficient NOG (NOG-dKO; NOG- lab, B2m-double-knockout) mice showed sufficient engraftment of human immune cells with little sign of GVHD. Immunization with influenza vaccine resulted in an increase in influenza-specific human IgG Ab, indicating induction of antigen-specific B cells in the NOG-dKO mice. Immunization with human dendritic cells pulsed with HLA-A2 restricted cytomegalovirus peptide induced specific cytotoxic $T$ cells, indicating the induction of antigen-specific T cells in the NOG-dKO mice. Adoptive cell therapies (ACTs) using melanoma antigen recognized by T cells (MART-1)-specific TCR-transduced activated T cells showed strong tumor growth inhibition in NOG-dKO mice without any sign of GVHD accompanied by preferential expansion of the transferred MART-1specific T cells. ACTs using cultured human melanoma infiltrating T cells also showed anti-tumor effects against autologous melanoma cells in NOG-dKO mice, in which changes in human cancer phenotypes by immune intervention, such as increased CD271 expression, could be evaluated. Therefore, NOG-dKO mice are useful tools for more detailed analysis of both the induction and effector phases of T-cell and B-cell responses for a longer period than regular NOG mice.

Cellular \& Molecular Immunology advance online publication, 20 November 2017; doi:10.1038/cmi.2017.106

Keywords: adoptive cell therapy; humanized mouse; vaccine

\section{INTRODUCTION}

Human in vivo immune responses are often presumed from mouse models because in vivo study of human biology is limited by ethical and technical problems. However, there are significant differences between humans and mice, such as T-cell and B-cell signaling pathway components, Th1/Th2 differentiation, Toll-like receptors and cytokines and their receptors. ${ }^{1}$ To overcome these problems, various humanized mice in which the human immune system can be studied in vivo have been recently developed. The most commonly used humanized mouse models are immunodeficient Il2ry null mouse strains, such as NOG (NOD/Shi-Prkdscid Il2r $\gamma^{\text {tml } 1 \text { Sug } / J i c)}$ mice $^{2}$ or NSG (NOD/LtSz-Prkdscid $I l 2 r \gamma^{\text {tm } 1 \text { Wjl } / \mathrm{J})} \quad$ mice $^{3}$ engrafted with human tissues, human hematopoietic cells (HSCs) or human peripheral blood mononuclear cells (PBMCs), in which human immune cells can be analyzed.

In conventional human HSC-engrafted NOG/NSG mice, human multi-lineage hematopoietic cells, including both

\footnotetext{
${ }^{1}$ Division of Cellular Signaling, Institute for Advanced Medical Research, Keio University School of Medicine, Tokyo 160-8582, Japan; ${ }^{2}$ Department of Dermatology, Yamanashi University School of Medicine, Yamanashi 409-3898, Japan; ${ }^{3}$ Department of Obstetrics and Gynecology, Keio University School of Medicine, Tokyo 160-8582, Japan and ${ }^{4}$ Central Institute for Experimental Animals, Kanagawa 210-0821, Japan

${ }^{5}$ These authors contributed equally to this work.

${ }^{6}$ Co-corresponding authors.

Correspondence: Professor Y Kawakami or Assistant Professor T Yaguchi, Division of Cellular Signaling, Institute for Advanced Medical Research, Keio University School of Medicine, 35 Shinanomachi, Shinjuku-ku, Tokyo 160-8582, Japan.
}

E-mail: yutakawa@keio.jp or beatless@rr.ij4u.or.jp

Received: 9 February 2017; Revised: 5 September 2017; Accepted: 6 September 2017 
lymphoid cells and myeloid cells, are developed from transplanted human HSCs, ${ }^{4}$ and these mice are widely used for human immunology research. However, these humanized mice are still insufficient because the developed human cells are not fully functional. For example, although immunization of these humanized mice with antigens leads to the production of antigen-specific IgM, antigen-specific IgG responses are very weak. ${ }^{5,6}$ T-cell differentiation, such as generation of CD4 and CD8 single-positive $\mathrm{T}$ cells, seems to be relatively normal in these humanized mice, ${ }^{5,7}$ However, the function of these $\mathrm{T}$ cells, especially induction of antigen-specific $\mathrm{T}$-cell responses, remains controversial. ${ }^{4}$ One of the reasons is thought to be mismatches between mouse MHC and HLA of donor human HSCs. To overcome these issues, new immunodeficient $I l 2 r \gamma^{\text {null }}$ mouse strains that express human HLA class I or class II molecules were generated and showed HLA-restricted antigenspecific CTL responses ${ }^{8}$ or antigen-specific IgG responses. ${ }^{9}$

Although these HSC-engrafted humanized mice have improved our understanding of in vivo human immune cell development and functions, ${ }^{10}$ these mice could not reconstruct the current immune status of human individuals, including patients, because all of the human hematopoietic cells were reconstituted from HSCs. In particular, analyzing the association of $\mathrm{T}$ cells and autologous tissues, including cancer cells, in HSC-engrafted humanized mice is nearly impossible because it is difficult to obtain HSCs and tissues from the same patient.

Human PBMC-engrafted humanized mice are attractive models for in vivo analysis of individual human patient immune responses. We previously reported that human PBMCs could be engrafted in NOG mice much better than in other immunodeficient mice. ${ }^{11}$ However, because of severe xenogeneic graft versus host disease (xeno-GVHD) in these mice, there is a limited window for experimentation. All the engrafted $\mathrm{T}$ cells are activated by xeno-GVHD, and all the mice die of xeno-GVHD, which makes it difficult to analyze human immune responses precisely. Previously, murine class I MHC and class II MHC-knockout NSG mice were developed and analyzed for xeno-GVHD, ${ }^{12}$ but the phenotypes and immune responses of transferred human PBMCs have not been clearly evaluated yet.

In this study, to overcome xeno-GVHD problems in the use of human PBMC-engrafted humanized mice that confound induced human immune responses, we have developed murine MHC class I- and class II-deficient NOG mice and analyzed whether these mice engrafted with human PBMCs could be useful tools for analysis of both the induction and effector phases of human T-cell and B-cell responses.

\section{MATERIALS AND METHODS}

Mice

NOD.Cg-Prkdscid Il2rg ${ }^{\text {tm1Sug} / J i c ~(N O D-s c i d ~ I L-2 r g ~}{ }^{\text {null: }}$ NOG)

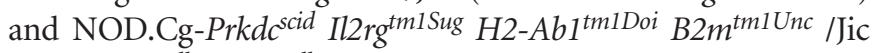
(NOG-Iab ${ }^{\text {null }} \mathrm{B}_{2} \mathrm{~m}^{\text {null: }}$ : NOG-dKO) mice were used in this study. NOG mice were established as previously reported. ${ }^{2}$ NOG-dKO mice were established as follows. B6;129-H2$\mathrm{Ab1}^{\text {tmlDoi }}\left(\mathrm{Iab}^{\text {null }}\right)$ and NOD.Cg-Prkdscid B2m $\mathrm{m}^{\text {tmlUnc/SzJ }}$
(NOD-scid B2m ${ }^{\text {null }}$ ) mice were a kind gift from Dr Mathis D. of Harvard Medical School and Dr Shultz L. of The Jackson Laboratory (Bar Harbor, ME, USA), respectively. Iab ${ }^{\text {null }}$ mice were first backcrossed into C57BL/6JJic mice more than seven generations. The established B6-Iab ${ }^{\text {null }}$ mice were further backcrossed into NOG mice with a speed congenic technique combining a marker-assisted selection protocol and in vitro fertilization to obtain NOG-Iab ${ }^{\text {null }}$ mice. NOG-B2 $\mathrm{m}^{\text {null }}$ mice were also established by backcross-mating NOD-scid B2m ${ }^{\text {null }}$ to NOG mice to introduce the $I l 2 \mathrm{rg}$ gene. NOG-dKO mice were obtained by mating NOG-Iab ${ }^{\text {null }}$ and NOG-B2 $\mathrm{m}^{\text {null }}$ mice. For genotyping NOG-B2m ${ }^{\text {null }}$ mice and NOG-Iab ${ }^{\text {null }}$ mice, 3 different primers were used for detection of WT and KO genotypes. The primers used to detect the $\beta 2$ microglobulin $(\mathrm{B} 2 \mathrm{~m})$ gene and I-ab gene were as follows: $\mathrm{B} 2 \mathrm{~m}$ gene, PI $($ B2 $\mathrm{m}$ forward $)=5^{\prime}$-AGAAGCCCAAGAGTTTCCTCATGG-3', PII $($ B2m reverse WT $)=5^{\prime}$-AAAGCCAAGATGCAGTGTGTA GCG-3', and PIII (B2m reverse $\mathrm{Mu})=5^{\prime}$-ACGTGCATGGAT CTGCAACATGTC-3'; I-ab gene, PI (Iab forward) $=$ 5'-GAGTACGTGCGCTACGACAG-3', PII (Iab reverse WT) = 5'-AGAGGGCAGAGGTGAGACAG-3', and PIII (Iab reverse $\mathrm{Mu})=5^{\prime}$-TGCTCCTGCCGAGAAAGTAT $-3^{\prime}$. Genomic DNA was extracted from the tails of the mice. The PCR reaction conditions were as follows: $94{ }^{\circ} \mathrm{C} 5 \mathrm{~min} / 94^{\circ} \mathrm{C} 30 \mathrm{~s}-60^{\circ} \mathrm{C} 30 \mathrm{~s}-$ $72{ }^{\circ} \mathrm{C} 60 \mathrm{~s}$ (35 cycles) $/ 72{ }^{\circ} \mathrm{C} 10 \mathrm{~min}$. A 676 -bp band was detected for mutant $\mathrm{B} 2 \mathrm{~m}$ from the PI and PIII primer pair. A 488-bp band was detected for wild-type B2m from the PI and PII primer pair. A 587-bp band was detected for mutant Iab from the PI and PIII primer pair. A 335-bp band was detected for wild-type Iab from the PI and PII primer pair. The absence of MHC class I molecule expression in NOG strains $\left(\mathrm{H}-2 \mathrm{~K}^{\mathrm{d}}\right.$ and $\left.\mathrm{H}-2 \mathrm{D}^{\mathrm{b}}\right)$ on the surface of cells from NOG-dKO mice was confirmed by FACS (Supplementary Figure 1). Mouse care and all experimental procedures were performed under pathogen-free conditions in accordance with the established guidelines of Keio University and Central Institute for Experimental Animals. For generating human PBMCtransplanted mice, human PBMCs were collected from healthy volunteers with informed consent using Lymphoprep (AXISSHIELD PoC AS, Oslo, Norway) and were injected into mice intravenously $\left(5 \times 10^{6} /\right.$ mouse $)$.

\section{Cells}

A human melanoma cell line, 526mel, and C1R-A2 cells expressing a transfected genomic clone of HLA-A2.1 were obtained from the Surgery Branch of the National Cancer Institute, National Institutes of Health (Bethesda, MD). Mel2 is a melanoma cell line established from the same patients from which TIL2 cells were established as previously described. ${ }^{13}$ These cells were cultured in RPMI1640 (Invitrogen, Carlsbad, CA, USA) supplemented with $10 \%$ fetal bovine serum and antibiotics. All cell cultures were maintained in a $5 \% \mathrm{CO}_{2}$ incubator at $37^{\circ} \mathrm{C}$. 


\section{Flow cytometry}

Peripheral blood cells from hPBMC-NOG mice were stained with fluorescein-conjugated monoclonal antibodies (Abs) for $1 \mathrm{~h}$. After red blood cells were lysed using OptiLyse C (Beckman Coulter Inc., Brea, CA, USA), the stained cells were analyzed using Gallios (Beckman Coulter Inc.). The antibodies used in this study were as follows; PE-Cy7 or Alexa Flour 700 anti-human CD45 (HI30), PE or PE-Cy7 anti-human CD8 (RPA-T8), FITC anti-human CD4 (RPA-T4), Horizon V450 anti-human CD3 (UCHT1), FITC anti-human CD19 (HIB19), Alexa Fluor 700 anti-human HLA-DR (G46-6), PE-Cy7 antihuman CD14 (M5E2), APC anti-human CD27 (M-T271), PECy7 anti-human CD10 (HI10a), Horizon V450 anti-human CD11c (B-ly6), PE anti-H-2K ${ }^{\mathrm{d}}$ (SF1-1.1), Alexa Flour 700 antiH-2D ${ }^{\mathrm{b}}$ (KH95), V500 or PerCP-Cy5.5 anti-mouse CD45 (30F11), FITC anti-human CD45RA (L48), PE/Dazzle 594 antihuman CD62L (DREG-56).

\section{Immunohistochemistry}

All tissue samples were fixed in 10\% neutral buffered formalin and embedded in paraffin. After deparaffinization, the sections were incubated in 3\% hydrogen peroxide for $30 \mathrm{~min}$ at room temperature and pretreated with HistoVTone (Nacalai Tesque, Kyoto, Japan) for $20 \mathrm{~min}$ at $90^{\circ} \mathrm{C}$. Then, after being blocked in $1 \%$ goat serum, the specimens were incubated with antihuman CD3 Ab (F7.2.38; Dako Japan, Tokyo, Japan) at $4{ }^{\circ} \mathrm{C}$ overnight. An avidin-biotin complex method was performed with a VECTASTAIN ABC Human IgG Kit (Vector laboratories, Burlingame, CA, USA), according to the manufacturer's instructions. The sections were developed with DAB in $0.003 \%$ hydrogen peroxide (Muto Pure Chemicals, Tokyo, Japan) and counterstained with hematoxylin. To determine the number of $\mathrm{CD}^{+} \mathrm{T}$ cells, five fields at $\times 400$ magnification per specimen were randomly chosen, and the positive counts were averaged.

\section{Influenza vaccination}

Influenza hemagglutinin (HA) vaccine (2010-2011 season; Kitasato Institute, Saitama, Japan), which was clinically used for humans, was obtained from the hospital pharmacy. Briefly, $5 \mu \mathrm{g}$ of HA was intraperitoneally administered one day after human PBMC transplantation (day 0). The anti-HA IgG titer in the sera of mice was measured on day 7 and day 14 by ELISA. On day 16, anti-HA IgG-producing B cells were examined with enzyme-linked immunospot assay (ELISPOT).

\section{ELISA}

The total human IgG in mouse sera was measured with a Human IgG ELISA Kit (Abcam, Cambridge, UK), according to the manufacturer's instructions.

The relative concentration of anti-HA antibodies in mouse sera was measured by ELISA. Influenza HA vaccine (2010-2011 season; Kitasato Institute, Saitama, Japan) diluted in PBS (5 $\mu \mathrm{g} /$ $\mathrm{ml}$ ) was coated on a 96-well micro-immune plate (Nunc, Denmark; $100 \mu \mathrm{l}$ per well) for $2 \mathrm{~h}$ at room temperature. After three washes with PBS, the plate was blocked with 5\% FBS/PBS (150 $\mu$ l per well) for $2 \mathrm{~h}$ at room temperature. Sample sera were diluted from $1: 100$ to $1: 218700$ by threefold serial dilution with $5 \%$ FBS/PBS. After being blocked, the plate was washed with PBST $(0.1 \%$ Tween 20/PBS) three times. Then, the serially diluted sample sera and standard serum $(100 \mu \mathrm{l}$ per well) were incubated for $2 \mathrm{~h}$ at room temperature. After three washes with PBST, the samples were incubated with horseradish peroxidase (HRP)-conjugated goat anti-human IgG (Southern Biotechnology Associates, Birmingham, AL, USA; 1:5000 in 5\% FBS/PBS, $100 \mu \mathrm{l}$ per well) for $1 \mathrm{~h}$ at room temperature. HRP activity was visualized using tetramethyl benzidine-free base (Sigma, St Louis, MO, USA) and phosphate-citrate buffer. The relative concentrations of anti-HA antibodies were estimated by comparison to standard curves on each plate. Serum from a healthy volunteer with anti-HA IgG was used as standard serum. The titer of standard sera was defined as $1000 \mathrm{U} / \mathrm{ml}$.

\section{ELISPOT}

Splenocytes from NOG-dKO mice were cultured $\left(0.5 \times 10^{6}\right.$ cells per $200 \mu \mathrm{l}$ per well) for $2 \mathrm{~h}$ in a 96-well plate with a nitrocellulose base (Millititer HA; Millipore, Bedford, MA, USA) coated with influenza HA vaccine (2010-2011 season; Kitasato Institute) diluted in PBS $(5 \mu \mathrm{g}$ per $100 \mu \mathrm{l}$ per well). After cells were removed, the plates were washed with $\mathrm{H}_{2} \mathrm{O}$ five times and incubated with $10 \mathrm{mM}$ EDTA-2Na/PBS $(150 \mu \mathrm{l}$ per well) for $10 \mathrm{~min}$ at room temperature. Then the plates were washed with PBST three times and PBS one time and incubated with $1 \%$ BSA/PBS at $4^{\circ} \mathrm{C}$ overnight. Then, the plates were overlaid with an alkaline phosphatase-labeled antihuman IgG1 detection antibody (Southern Biotech, Birmingham, AL, USA) for $1 \mathrm{~h}$ at room temperature. After a wash with PBS, a precipitating substrate for alkaline phosphatase was added, and the plates were incubated until spots emerged at the site of the responding cells. The spots were counted using a CTL-ImmunoSpot S5 Micro Analyzer (Cellular Technology Limited, Shaker Heights, OH, USA).

\section{Multiplex cytokine analysis}

Various cytokines in sera from hPBMC-NOG-dKO mice and hPBMC-NOG mice were measured 23 days after human PBMC implantation using a Bio-plex pro human cytokine Grp 1 panel and Grp 2 panel (Bio-rad, Hercules, CA, USA), according to the manufacturer's instructions.

\section{Dendritic cell vaccination}

Human monocyte-derived DCs (Mo-DCs) were generated as previously reported. ${ }^{14}$ Briefly, human $\mathrm{CD} 14^{+}$monocytes were isolated from human PBMCs using MACS beads (Miltenyi Biotec, Bergisch Gladbach, Germany) and cultured with DC medium containing RPMI1640, 1.5\% human autologous sera, GM-CSF $(100 \mathrm{ng} / \mathrm{ml})$ and IL-4 $(50 \mathrm{ng} / \mathrm{ml})$ for 2 days (1.5$2 \times 10^{7}$ cells $/ 10 \mathrm{~cm}$ dish). Then, $10 \mathrm{ng} / \mathrm{ml} \mathrm{TNF-} \alpha, 10 \mathrm{ng} / \mathrm{ml}$ IL-1 $\beta, 250 \mathrm{ng} / \mathrm{ml}$ PGE2, $5000 \mathrm{U} / \mathrm{ml}$ IFN- $\gamma, 20 \mathrm{ng} / \mathrm{ml}$ poly(I:C) and $1 \mathrm{mg} / \mathrm{ml} \mathrm{R848}$ were added for cell maturation. On day 3, the Mo-DCs were harvested and pulsed with HLA-A2 restricted CMVpp65495-503 peptide (NLVPMVATV; $5 \mu \mathrm{g} / \mathrm{ml}$ in $1 \mathrm{ml}$ of AIM-V medium) for $1 \mathrm{~h}$. Then, these $3-5 \times 10^{6}$ 
pulsed Mo-DCs were intravenously administered to human PBMC-transferred NOG-dKO mice. This DC vaccine was administered on day 0 , day 7 and day 14 . Unpulsed Mo-DCs were administered to the control group. On day 21, human $\mathrm{T}$ cells were harvested from the spleen, peripheral blood, and bone marrow of vaccinated-NOG-dKO mice using CD3 MACS beads (Miltenyi Biotec). The sorted human T cells $\left(1.5 \times 10^{6}\right.$ per well per $2 \mathrm{ml}$ ) were cocultured with irradiated $(32 \mathrm{~Gy}) \mathrm{CD}^{+} \mathrm{T}$ cell-depleted autologous PBMCs $\left(3 \times 10^{6}\right.$ per well per $\left.2 \mathrm{ml}\right)$ in a 24-well plate with CMVpp65 peptide $(2.5 \mu \mathrm{g} / \mathrm{mL})$, IL-2 (20 U/ $\mathrm{ml})$, and IL-7 $(10 \mathrm{ng} / \mathrm{mL})$ for 7 days. Then, T cells were harvested using Lymphoprep (AXIS-SHIELD PoC AS) and stained with PerCP-Cy5.5 anti-CD45, PE-Cy7 anti-CD8, brilliant violet anti-CD3, and PE HLA-A*02:01 CMV pp65 tetramer-NLVPMVATV (MBL, Nagoya, Japan). PE HLA$\mathrm{A}^{\star}$ 02:01 HIV gag tetramer-SLYNTVATL (MBL) was used as a negative control.

\section{Generation of MART-1-specific T cells}

MART-1-specific T cells (MART-Ts) were generated as previously described. ${ }^{15}$ Briefly, the plasmid encoding the HLA$A^{\star}$ 0201-restricted MART-1-specific TCR was a gift from Dr Ken-ichi Hanada (Surgery Branch, National Cancer Institute, National Institutes of Health, USA). PBMCs from healthy donors were stimulated with soluble OKT-3 (50 ng/ml) and recombinant human IL-2 (300 IU/ml; Novartis, East Hanover, a

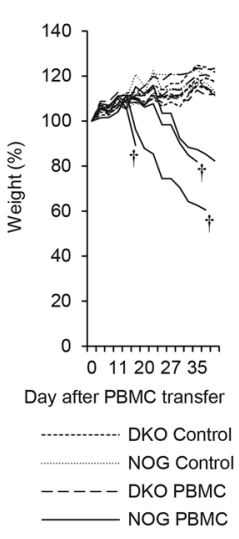

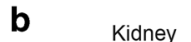
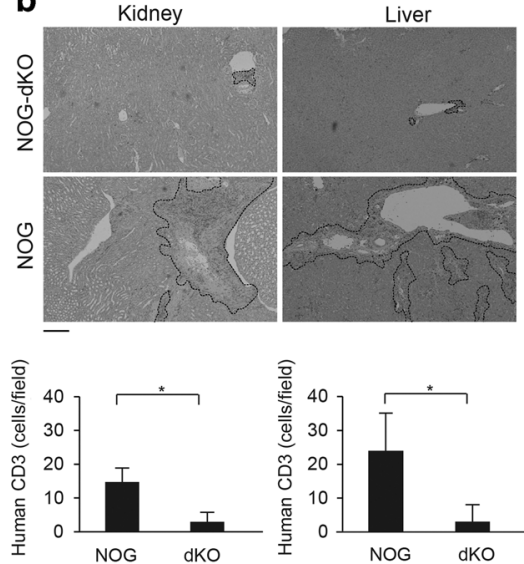

Figure 1 Mild GVHD in human peripheral blood mononuclear cell (PBMC)-transplanted murine $\mathrm{MHC}$ class I- and class II-deficient NOG mice. (a) Body weight changes in NOG and NOG-dKO mice with or without intravenous transplantation of human PBMCs are shown. $\dagger$ indicates death of the mouse. Only human PBMCtransferred NOG mice showed severe body weight loss, finally dying of severe graft versus host disease (GVHD). \% weight indicates the percentage weight change from the initial weight. Each line indicates an individual mouse $(n=4-5)$. (b) Twenty-eight days after human PBMC transplantation, human $\mathrm{CD}^{+} \mathrm{T}$ cells infiltrating into the kidney and liver (area indicated by the dotted line) were analyzed via immunohistochemistry. For calculating $\mathrm{CD}^{+} \mathrm{T}$ cells, five fields at $\times 400$ magnifications per specimen were randomly chosen, and the positive counts were averaged. Bars represent $200 \mu \mathrm{m}$. The data are representative of three experiments. Error bars are \pm s.d.; ${ }^{*} P<0.05$ ( $t$-test).
NJ, USA) for 2 days before transduction. The stimulated cells were then transfected with a retrovirus expressing the MART-1-specific TCR gene. The specificity of the generated MART-Ts was evaluated by determining the production of IFN- $\gamma$ from MART-Ts cocultured with HLA-A2 epitope peptide (gp100 $154-162$ : KTWGQYWQV or MART-1 $27-35$ : ELAGIGILTV)-pulsed C1R-A2 cells.

\section{In vitro expansion of human melanoma TILs}

TIL2 cells were cultured tumor infiltrating lymphocytes established from the same patient from which Mel2 cells were established, as described previously. ${ }^{13}$ Briefly, melanoma tumor digests were cultured in a 24-well plate containing $2 \mathrm{ml}$ of RPMI1640 supplemented with 10\% human serum, antibiotics and recombinant human IL-2 (6000 IU/ml, Novartis). Five days after initiation, half of the medium was replaced with fresh complete medium containing IL-2; this was repeated every 2 to 3 days thereafter as needed. The recognition of Mel2 cells by TIL2 cells was confirmed by examining IFN- $\gamma$ production from TIL2 cells $\left(2 \times 10^{5}\right)$ cocultured with Mel2 cells $\left(1 \times 10^{5}\right)$ with $10 \mu \mathrm{g} / \mathrm{ml}$ of anti-HLA-ABC Ab (W6/32, BioXcell, West Lebanon, NH, USA) or mouse IgG2a isotype control (C1.18.4, BioXcell).

\section{Adoptive cancer immunotherapy models}

Briefly, $5 \times 10^{6} 526 \mathrm{mel}$ cells were subcutaneously inoculated into the flank of NOG or NOG-dKO mice. Cultured MART-Ts $\left(5 \times 10^{6}\right.$ cells per mouse) were intravenously administered on day $9.4 \times 10^{6} \mathrm{Mel} 2$ cells were subcutaneously inoculated into the flank of NOG-dKO mice. Cultured TIL2 cells were intraperitoneally administered on day $0\left(3.75 \times 10^{7}\right)$ and day $3\left(1 \times 10^{8}\right)$. Tumor volumes were calculated according to the following formula: [(length $\left.) \times(\text { width })^{2}\right] / 2$.

\section{RESULTS}

Mild GVHD in human PBMC-transplanted murine MHC class I- and class II-deficient NOG mice

Murine MHC class I- and class II-deficient NOG (NOD/Shiscid IL2rg ${ }^{\text {null }}$ ) mice (NOG-dKO) were generated by mating $\beta 2$-microgrobulin-knockout NOG mice (NOG-B2m ${ }^{\text {null }}$ ) and Iab-knockout NOG mice (NOG-Iab ${ }^{\text {null }}$ ). To verify the usefulness of NOG-dKO mice as a mouse model enabling long-term analysis of immune responses by transplanted human peripheral immune cells, we compared the onset and symptoms of xeno-GVHD between NOG mice and NOG-dKO mice in which $5 \times 10^{6}$ human PBMCs were transplanted. NOG mice implanted with human PBMCs (hPBMC-NOG) died of severe xeno-GVHD within 2 to 6 weeks with a reduction in body weight (Figure 1a) and with human T-cell infiltration into various organs, including the kidney, liver, colon, skin, lung and spleen, while NOG-dKO mice implanted with human PBMCs (hPBMC-NOG-dKO) survived for $>4$ months without significant body weight loss and with less human T-cell infiltration in the various organs (Figures $1 \mathrm{a}$ and $\mathrm{b}$ and Supplementary Figure 2). We also measured various human cytokines in the mouse sera on day 23 using Bio-plex and 
a

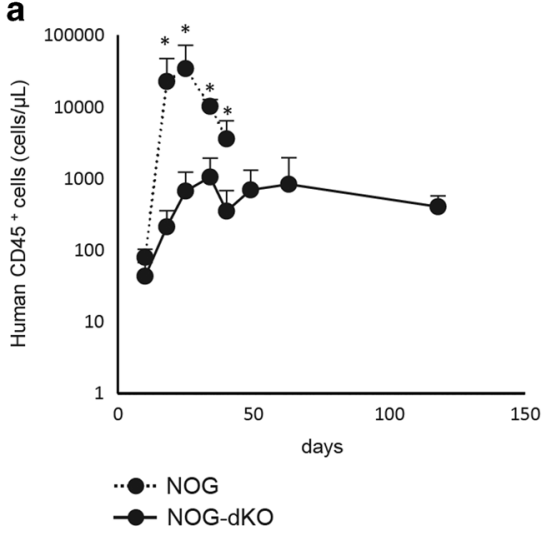

d

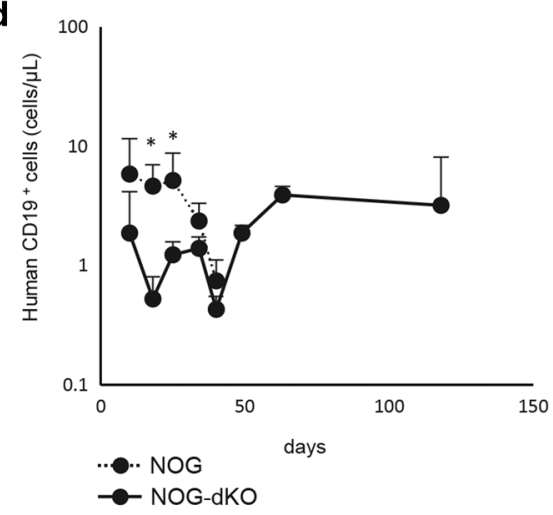

b

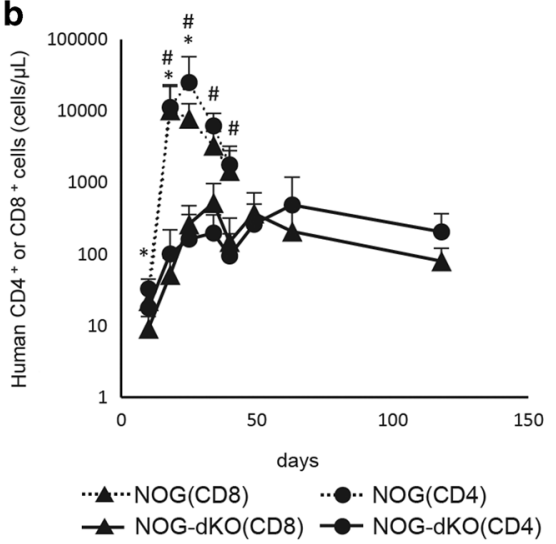

C

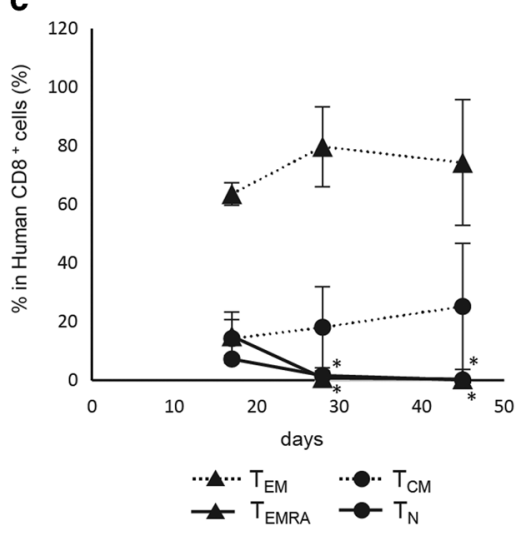

e

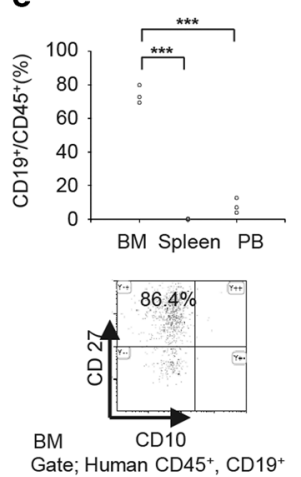

f

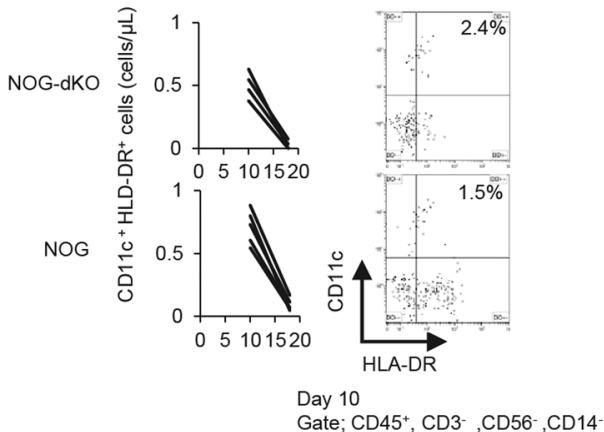

Figure 2 Analysis of transferred human PBMCs in NOG-dKO mice. (a, b, $\mathbf{d}$ and $\mathbf{f}$ ) After intravenous transplantation of human PBMCs into

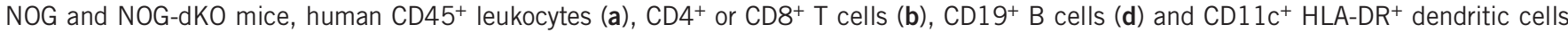

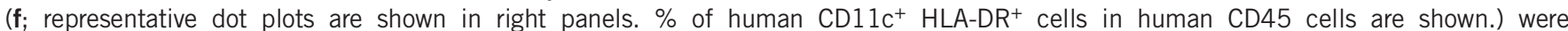
monitored in the peripheral blood of the mice using FACS. Human T cells and B cells could be detected for $>100$ days in the NOG-dKO

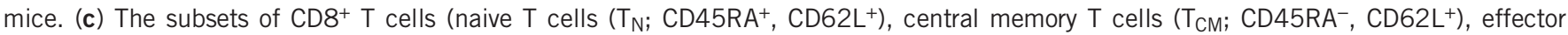
memory $T$ cells $\left(T_{E M} ; C D 45 R A^{-}, C_{2} 6 L^{-}\right)$, and terminally differentiated effector memory cells re-expressing CD45RA ( $T_{E M R A}$; $C D 45 R A^{+}$, $\left.\mathrm{CD}_{2} \mathrm{~L}^{-}\right)$) are shown. (e) Human $\mathrm{CD} 19^{+} \mathrm{B}$ cells were analyzed in the bone marrow (BM), spleen, and peripheral blood (PB) on day 83 (top panel). The expression of B-cell differentiation markers on B cells in the BM from a representative mouse (bottom panel). The data are representative of 5 experiments. $n=4-5$. \# or * indicates $P<0.05$ (the numbers of the indicated cells in NOG mice were significantly higher than those in NOG-dKO mice at each day marked with * or \# (a, b and $\mathbf{d}) .{ }^{*}$ is for CD8 and \# is for CD4 (b). The numbers of the indicated cells on d28 or d45 was significantly lower than those on d17 (c). A t-test was applied on the logarithmic scale (a, b and $\mathbf{d})$ and on the linear scale (c)).

found that IFN- $\gamma(\mathrm{NOG}-\mathrm{dKO} ; 910.1 \pm 986.7 \mathrm{pg} / \mathrm{ml}, \mathrm{NOG}$; $4557.8 \pm 1348.1 \mathrm{pg} / \mathrm{ml} \quad(P<0.005 ; \quad t$-test $) \quad$ and RANTES $(\mathrm{NOG}-\mathrm{dKO} ; 281.4 \pm 284.2 \mathrm{pg} / \mathrm{ml}$, NOG; $1423.5 \pm 196.4 \mathrm{pg} / \mathrm{ml}$ $(P<0.001 ; t$-test $))$, which are known to be related to T-cell activation, were significantly lower in the sera of NOG-dKO mice. These results suggest that the NOG-dKO mouse might be useful as a mouse model for analyzing transferred human PBMCs with less xeno-GVHD effects.

\section{Engraftment of transferred human PBMCs in NOG-dKO} mice

$\mathrm{CD}^{+} \mathrm{T}$ cells and $\mathrm{CD}^{+} \mathrm{T}$ cells are the major populations in transferred human PBMCs expanded in NOG mice and NOG$\mathrm{dKO}$ mice (Figures 2a and $\mathrm{b}$ ). Transferred human $\mathrm{T}$ cells increased more moderately in NOG-dKO mice than in NOG mice but could be detected for at least 4 months (Figure $2 b$ ).
The major populations of $\mathrm{CD}^{+} \mathrm{T}$ cells that expanded in NOG$\mathrm{dKO}$ mice were central memory $\mathrm{T}$ cells and effector memory $\mathrm{T}$ cells (Figure $2 \mathrm{c}$ ). Although the number of $\mathrm{B}$ cells in the peripheral blood is relatively low, they were detected for at least 4 months (Figure 2d), and human IgG was also detected in sera $(68.0 \pm 21.1 \mu \mathrm{g} / \mathrm{ml}$ on day $23,194.9 \pm 177.4 \mu \mathrm{g} / \mathrm{ml}$ on day 71$)$. Compared with the spleen and peripheral blood, the bone marrow contained a much higher number of $\mathrm{B}$ cells with memory B-cell phenotypes $\left(\mathrm{CD} 10^{-}, \mathrm{CD} 27^{+}\right)$on day 83, suggesting possible memory $\mathrm{B}$ cell homing to the bone marrow (Figure 2e). While T cells and B cells were detected for at least 4 months in NOG-dKO mice, dendritic cells (CD11c ${ }^{+}$HLA$\mathrm{DR}^{+}$cells) were detected for only 10 days (Figure $2 \mathrm{f}$ ). These results indicate that human $\mathrm{B}$ cells, $\mathrm{T}$ cells and antigen presenting cells can be engrafted in NOG-dKO mice with little sign of GVHD. 
Induction of antigen-specific B-cell responses by influenza vaccination in human PBMC-transferred NOG-dKO mice Next, we evaluated whether antigen-specific B-cell responses can be induced in the hPBMC-NOG-dKO mice. After human PBMCs were transferred to the NOG-dKO mice from healthy human volunteers who had anti-influenza hemagglutinin (HA) IgG antibodies in their sera, the mice were intraperitoneally immunized with HA vaccine. Then, 14-16 days after the vaccination, an increase in human HA-specific IgG Ab (Figure 3a) and HA-specific IgG production from the transferred human B cells (Figure 3b) were observed in the sera and spleen of the NOG-dKO mice, respectively. These results suggest that antigen-specific B-cell responses can be induced by immunization of the antigen in hPBMC-NOG-dKO mice.

\section{Induction of antigen-specific $\mathrm{T}$-cell responses by dendritic cell vaccination in human PBMC-transferred NOG-dKO mice}

Next, we evaluated whether antigen-specific T-cell responses could be induced in the hPBMC-NOG-dKO mice. Because of poor survival of antigen presenting cells, including dendritic cells, in the hPBMC-NOG-dKO mice (Figure 2f), we repeatedly immunized the mice with antigen-pulsed autologous mature monocyte-derived dendritic cells (Mo-DCs) for induction of antigen-specific $\mathrm{T}$ cells. After human PBMCs from healthy volunteers who had memory CMV-specific $\mathrm{CD}^{+}$ $\mathrm{T}$ cells were transferred into the NOG-dKO mice, we weakly immunized the mice with autologous Mo-DCs pulsed with HLA-A0201-binding CMV antigen-derived peptide (CMVpp65495-503; NLVPMVATV) three times. After these DC immunizations, human $\mathrm{CD}^{+} \mathrm{T}$ cells were isolated from the spleen, peripheral blood and bone marrow of the immunized hPBMC-NOG-dKO mice on day21, re-stimulated with CMVpp65 $495-503$ peptide for a week in vitro, and evaluated for the induction of CMVpp65-specific T cells using an HLA0201/ CMVpp65 $495-503^{-t e t r a m e r}$ (Figure 4a). CMVpp65-specific T-cell induction was observed in the spleen, bone marrow,
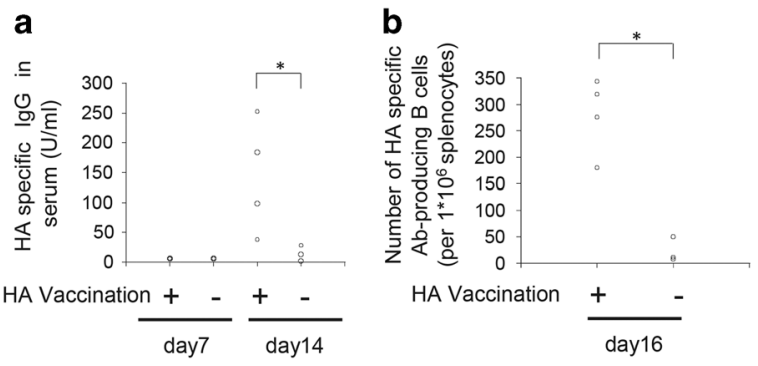

Figure 3 Influenza HA-specific B-cell responses were induced by $\mathrm{HA}$ vaccination in the human PBMC-transferred NOG-dKO mice. NOG-dKO mice were intraperitoneally immunized with influenza HA vaccine on the day after intravenous transplantation of human PBMCs. On day 7 and day 14, mice sera were collected and the anti-HA IgG titer was measured by ELISA (a). On day 16, the number of anti-HA IgG-producing human B cells collected from mouse spleens were measured by ELISPOT (b). The data are representative of three experiments. Each dot indicates an individual mouse $(n=3-4) .{ }^{*} P<0.05$ (t-test). and peripheral blood of the DC-immunized mice (Figure 4b), indicating that the induction of antigen-specific $\mathrm{T}$ cells can be evaluated in the hPBMC-NOG-dKO mice.

Adoptively transferred tumor-specific T cells can show antitumor effects with little sign of GVHD in NOG-dKO mice NOG mice have been used previously for evaluation of the in vivo killing activity of adoptively transferred human tumorspecific T cells. However, severe GVHD has been an obstacle for long-term follow-up and precise evaluation of the function of the $\mathrm{T}$ cells. We evaluated whether NOG-dKO mice can be used as a mouse model for T-cell-based adoptive cancer immunotherapies. We implanted 526 mel, a human melanoma cell line expressing MART- $1,{ }^{16}$ a human melanoma antigen, and HLA-0201, in NOG mice and NOG-dKO mice and treated them with MART-1-specific T cells (MART-Ts), which were generated by transducing the HLA-0201-restricted MART-1specific TCR gene into activated PBMC-derived T cells from healthy human volunteers ${ }^{15}$ (Figure 5a). The NOG-dKO mice showed anti-tumor effects induced by MART-T administration with no body weight loss (Figures $5 \mathrm{~b}$ and c). Co-administration of exogenous human recombinant IL-2 prolonged the survival of T cells with higher MART-1/HLA-A2 tetramer-positive cells and enhanced the anti-tumor effects in NOG-dKO mice (Figures $5 \mathrm{~d}$ and e). Although the NOG mice showed stronger anti-tumor effects along with increased transfer of $\mathrm{T}$ cells, probably due to strong T-cell expansion via xenogeneic GVHD responses, the ratio of MART-1-specific $\mathrm{T}$ cells in the $\mathrm{CD}^{+}$ $\mathrm{T}$ cells was smaller than that in NOG-dKO mice, suggesting that T-cell phenotypes were modified by xenogeneic GVHD responses in NOG mice (Figure 5e). Next, we treated human melanoma-bearing NOG-dKO mice with autologous tumor infiltrating lymphocytes (TILs). In NOG-dKO mice, administration of cultured tumor infiltrating $\mathrm{T}$ cells, TIL2 ${ }^{17}$ cells (Figure 5f), showed significant anti-tumor effects against an autologous melanoma cell line, Mel2, which was established from the same patient from which the TIL2 cells were established (Figure 5g). CD271 on human melanoma cells, which is reported to be induced by IFN- $\gamma$ and involved in suppression of melanoma-specific CTLs, ${ }^{18}$ was found to be increased in TIL2-treated melanoma cells (Figure 5h), suggesting that NOG-dKO mice might also be used for evaluation of changes in human cancer phenotypes induced by T-cells following immune interventions. Altogether, the results of this study demonstrated that NOG-dKO mice are more useful for detailed and long-term analysis of B-cell responses and $\mathrm{T}$ cell responses at both the induction and effector phases of human peripheral blood than regular NOG mice.

\section{DISCUSSION}

Human PBMC-engrafted immunodeficient mice might be good models for analyzing human individual immune status, such as B-cell responses to vaccination and $\mathrm{T}$-cell responses against autologous cancers. However, severe xeno-GVHD has been a formidable obstacle for long term and precise evaluation of human immune responses induced in these mice. In this 
a

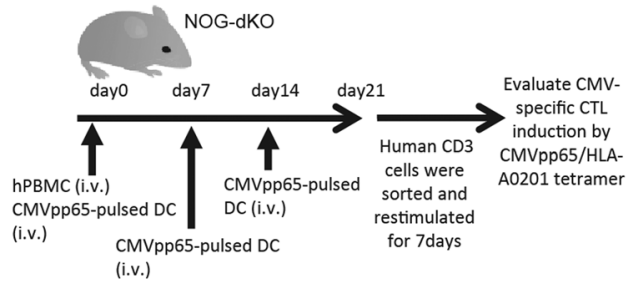

b

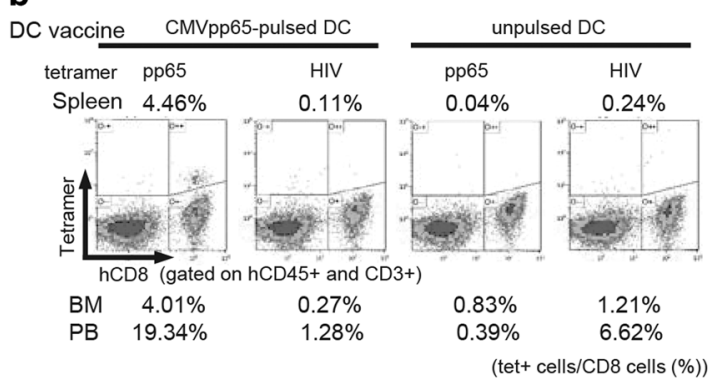

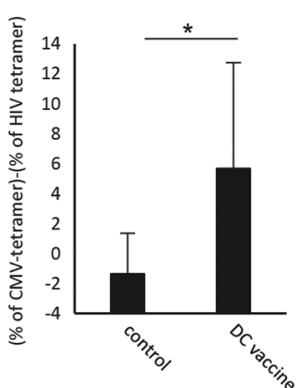

Figure 4 CMV-specific T-cell responses were induced by dendritic cell vaccines in the human PBMC-transferred NOG-dKO mice. Schema of the experiment (a). Human PBMC-transferred NOG-dKO mice were weekly immunized with HLA-0201-binding peptide (CMVpp65495503)-pulsed autologous monocyte-derived DCs (Mo-DCs) three times. Control mice were immunized with un-pulsed Mo-DCs. On day 21, human $\mathrm{CD}^{+} \mathrm{T}$ cells were sorted from the peripheral blood (PB), spleen, and bone marrow (BM) and re-stimulated with irradiated CMVpp65 peptide-pulsed CD3-depleted autologous hPBMCs in vitro for 7 days. Then, CMV-specific CTL induction was evaluated using HLA0201/CMVpp65495-503-tetramer. HLA0201/HIVgag77-85-tetramer was used as negative control. The values are \% of tetramer-positive cells in $\mathrm{CD}^{+}$positive cells. The data are representative of two experiments (b, left). The CMV-specific CTL induction was calculated as (\% of CMV-tetramer) - (\% of HIV tetramer), and the averaged CTL induction in the spleen, BM, and PB from two independent experiments was compared between the DC vaccine group and control group. ${ }^{*} P<0.05$; Mann-Whitney test (b, right).

study, we newly established murine MHC class I- and class IIdeficient NOG mice and showed much milder GVHD induced by transferred human $\mathrm{T}$ cells and success of the induction of human B-cell and T-cell immune responses in hPBMC-NOGdKO mice.

Generally, NOG/NSG mice, which additionally lack NK activities, showed significant improvement in survival of engrafted human PBMCs and in xeno-GVHD induction compared with other immunodeficient mouse models, such as NOD/SCID mice. ${ }^{4}$ King et al. ${ }^{19}$ have reported that the development of xeno-GVHD in NSG mice is highly dependent on host expression of both murine MHC class I and class II molecules. They have shown that both MHC class I-singleknockout mice and MHC class II-single-knockout mice developed xeno-GVHD. In this study, double-knockout of both murine MHC class I and class II molecules results in much milder xeno-GVHD in hPBMC-NOG-dKO mice, suggesting that together the MHC class I and MHC class II molecules in recipients play a major role in the pathogenesis of GVHD.

Although there was no obvious symptomatic sign of GVHD in hPBMC-NOG-dKO mice, the number of human $\mathrm{T}$ cells in hPBMC-NOG-dKO mice continued to increase until three weeks after PBMC engraftment, and mild human T-cell infiltration was observed in their organs (Figures $1 \mathrm{~b}$ and $2 \mathrm{a}$ and Supplementary Figure 2). One possible mechanism is incomplete downregulation of MHC class I molecules by the deletion of the $\beta 2$ microglobulin gene, although we confirmed little expression of MHC class I molecules on the surface of cells from NOG-dKO mice (Supplementary Figure 1). The other possible mechanisms involved in the stimulation of engrafted human $\mathrm{T}$ cells might be homeostatic proliferation, engrafted human antigen-presenting cells presenting murine proteins, and murine minor histocompatibility antigens (mHAs). Further analysis is needed to elucidate the mechanism underlying this T-cell stimulatory effect.
B-cell responses in human PBMC-engrafted immunodeficient mice have been previously reported. For example, Okada et al. ${ }^{20}$ reported that vaccination of cDNA constructs encoding the antigenic protein of severe acute respiratory corona virus induced the production of Ag-specific antibodies. However, the B-cell response in these mice might be influenced by xenoGVHD reaction. In this study, we showed influenza HAspecific IgG production in hPBMC-NOG-dKO mice vaccinated with HA antigen, suggesting that even with little inflammation caused by xeno-GVHD reaction, B-cell responses can be induced. Lack of xeno-GVHD in our mouse models enables more long-term and precise evaluation of human B-cell characteristics and responses. For example, the observation of $\mathrm{B}$ cells homing to the bone marrow (BM) almost 3 months (long-term) after human PBMC transfer in NOG-dKO mice (Figure 2e) is of interest. The mechanism of human B-cell homing to the BM and generation of memory $\mathrm{B}$ cells could be analyzed using our mouse models.

Considering the lack of de novo multi-lineage human hematopoiesis, it seems to be difficult to induce primary immune responses in human PBMC-engrafted immunodeficient mouse models. ${ }^{10}$ Thus, the mechanism of IgG production in our mouse models might be related to reactivation of antigen-experienced memory B cells. Whether primary immune responses can be induced by supplying antigen presenting cells in our mouse models remains to be evaluated in the future.

Although the development of $\mathrm{CD}^{+}$and $\mathrm{CD} 8^{+} \mathrm{T}$ cells and reconstitution of human acquired immunity ${ }^{5,7,21,22}$ have been shown in human HSC-transplanted immunodeficient $I l 2 r \gamma^{\text {null }}$ mouse strains, the induction of human HLA-restricted T-cell responses in humanized mice was limited by the lack of HLA expression. Some groups reported that the introduction of human HLA expression improved human HLA-restricted CTL responses. ${ }^{8,23-26}$ These previous studies used human HSCs to reconstitute human immune systems, which are not suitable 


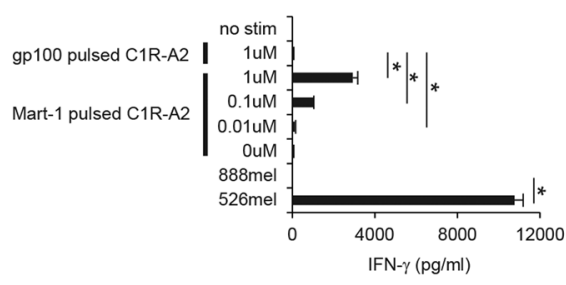

b

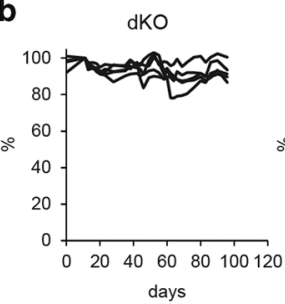

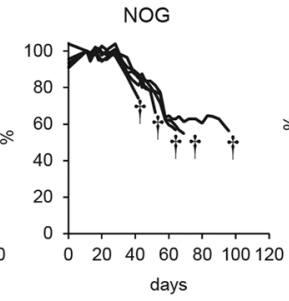

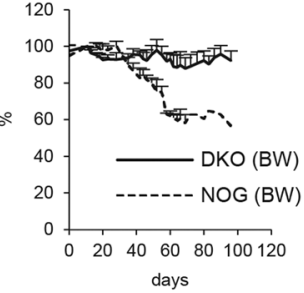

C
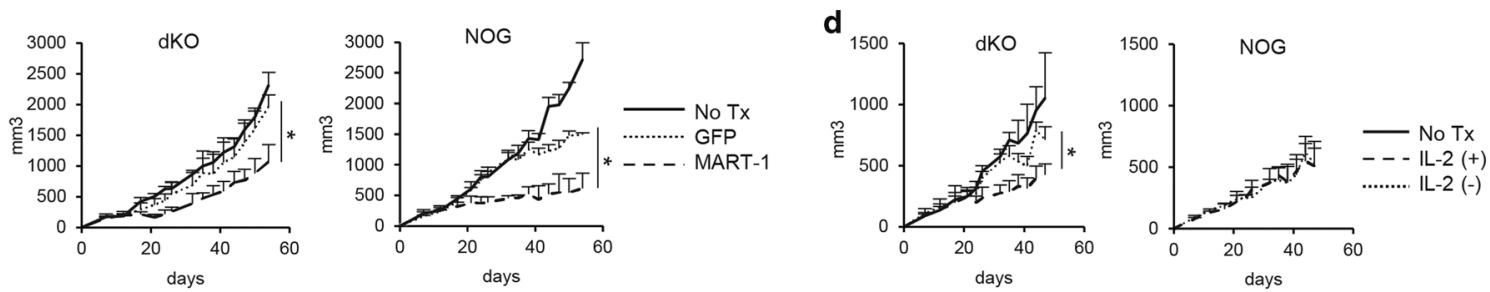

e
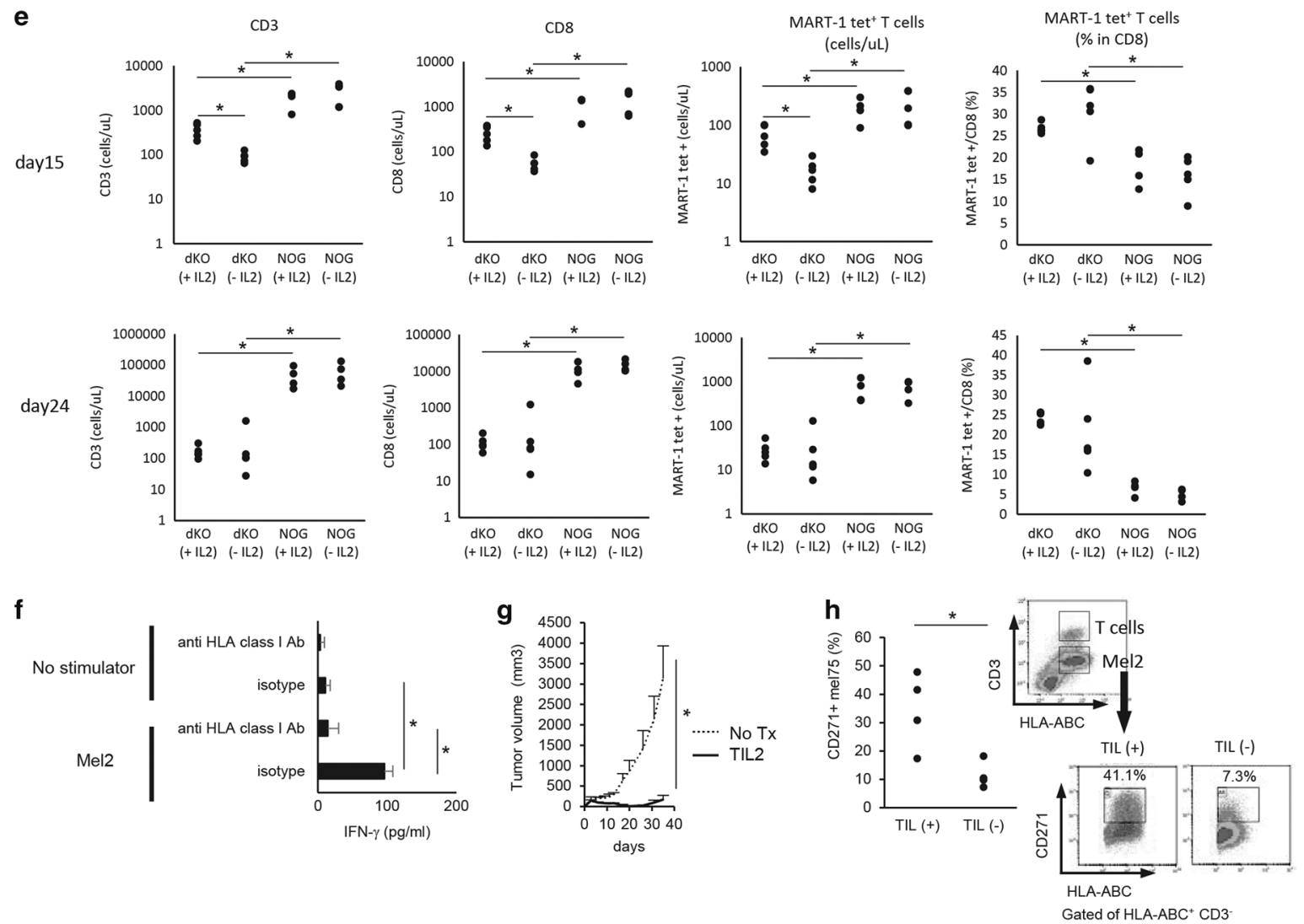

Figure 5 Adoptively transferred tumor-specific T cells can show anti-tumor effects with little sign of GVHD in NOG-dKO mice. (a) The MART-1-specific T cells (MART-Ts) used in the experiment specifically recognized MART-1 in an HLA-0201-restricted manner. MART-Ts were cocultured for $18 \mathrm{~h}$ with C1R-A2 cells pulsed with a HLA-0201-binding MART-1 peptide or an HLA-0201-binding gp100 peptide, 526 mel (an HLA-A*0201+ MART-1 ${ }^{+}$melanoma cell line) cells, or $888 \mathrm{mel}$ (an HLA-A*0201- melanoma cell line) cells. IFN- $\gamma$ in the supernatant was measured by ELISA. (b-e) $526 \mathrm{mel}$ cells were inoculated into NOG or NOG-dKO mice and treated with MART-Ts. Body weight changes (b) and tumor growth (c) in NOG and NOG-dKO mice are shown. $\dagger$ indicates death of the mouse. \% weight indicates the percentage weight change from the initial weight. The anti-tumor effects of MART-Ts in NOG-dKO mice were augmented by daily administration of recombinant human IL2 (5000 IU per mouse) (d). The absolute numbers of administered human $\mathrm{CD}^{+}$, $\mathrm{CD}^{+}$, or $\mathrm{HLA}^{-}$ A2/MART-1 $27-35_{\text {-tetramer }}{ }^{+}$cells and the $\%$ of HLA-A2/MART-1 $27-35$-tetramer ${ }^{+}$cells in CD8 ${ }^{+}$cells in the peripheral blood of NOG or NOGdKO mice were measured via FACS (e). $n=4-5$. (f) The TIL2 cells used in the experiment recognized autologous melanoma (Mel2) cells in an MHC class I-dependent manner. TIL2 and Mel2 cells were cocultured for $18 \mathrm{~h}$ with anti-HLA-ABC neutralizing Ab or its isotype control. IFN- $\gamma$ in the supernatant was measured by ELISA. (g) Mel2 cells were inoculated into NOG-dKO mice and treated with autologous TIL2 cells. $n=4$. (h) On day 35, CD271 expression on Mel2 cells was evaluated via FACS. Representative FACS dot plots are shown in the right panels. The data are representative of three $(\mathbf{a}-\mathbf{e})$ or two $(\mathbf{f}-\mathbf{h})$ experiments. Error bars are \pm s.d. ${ }^{*} P<0.05$ ( $t$-test). 
for analyzing human current immune status of individuals, including patients.

Chun et $a .^{27}$ previously reported that NOD/SCID $\beta_{2}$ microglobulin $^{-1-}$ mice engrafted with human HSCs and further reconstituted with autologous peripheral $\mathrm{T}$ cells could induce HLA-restricted T-cell responses against influenza virus vaccines. Spranger et al. ${ }^{28}$ previously reported that in humanPBMC engrafted NSG mice vaccination with human Mo-DCs expressing MART-1 could induce MART-1-specific $\mathrm{T}$ cells. These previous studies showed the induction of HLA-restricted T-cell responses from human peripheral blood; however, the influence of xeno-GVHD might be a problem for evaluation of these T-cell responses in these humanized mice. In this study, we showed that immunization with human Mo-DCs pulsed with HLA-A2 restricted CMV peptides induced CMV-specific CTLs in hPBMC-NOG-dKO mice. Our humanized mouse system showed that with little xenogeneic stimulation antigenspecific $\mathrm{T}$ cells could be induced, and this system may be an attractive model for more precise evaluation of human peripheral T-cell responses.

To evaluate the anti-tumor effect of human tumor-specific $\mathrm{T}$ cells, such as tumor infiltrating lymphocytes and genetically engineered human $\mathrm{T}$ cells expressing chimeric antigen receptors (CARs) ${ }^{29}$ or TCRs, ${ }^{30}$ previous studies often utilized human tumor-bearing immune deficient mice, such as NOG, NSG, and NOD/SCID mice. In these models, the xeno-GVHD reaction stimulates transferred $\mathrm{T}$ cells, alters their phenotypes and masks the real anti-tumor effects. Actually, in our experiment, transferred MART-1-specific T cells were preferentially expanded in NOG-dKO mice compared with NOG mice (Figure 5e). The effector phase of anti-tumor immune responses in which anti-tumor $\mathrm{T}$ cells recognize and kill tumor cells can be evaluated more precisely and for a longer period in our NOG-dKO mouse models than in NOG mouse models because of the lack of xeno-GVHD. In particular, T-cell phenotypes, such as anergy or exhaustion, might be effectively evaluated. Our colleagues have recently reported that immunotherapies targeting PD-1 could be more precisely evaluated in our NOG-dKO mice than in NOG mice because the xenoGVHD reaction induces nonspecific T-cell activation accompanied by PD-1 expression in NOG mice. ${ }^{31}$ On the other hand, because of the short longevity of antigen presenting cells, our current mouse models may not be suitable for evaluation of the induction phase of anti-tumor immune responses if DCs are not supplied, as shown in Figure 4. A variety of immune cells, such as macrophages, MDSCs, Tregs and NK cells, which play important roles in the cancer microenvironment, are also not well supported in our current mouse models. For supporting these cells, we are now developing various human cytokineproducing transgenic NOG strains. ${ }^{32}$

In conclusion, compared with NOG mice, hPBMC-NOG$\mathrm{dKO}$ mice are useful for analyzing current human individual immune status and providing long-term and more precise evaluation of human T-cell and B-cell responses.

\section{CONFLICT OF INTEREST}

The authors declare no conflict of interest.

\section{ACKNOWLEDGEMENTS}

This work was supported by Grants-in-aid for Scientific Research from the Ministry of Education, Culture, Sports, Science and Technology (MEXT) of Japan (22220007, 26221005 and 15K09783); the Project for Development of Innovative Research on Cancer Therapeutics (P-DIRECT) and the Project for Cancer Research And Therapeutic Evolution (P-CREATE) from Japan Agency for Medical Research and Development (AMED); a grant from Tokyo Biochemical Research Foundation; a Keio University Grant-in-Aid for Encouragement of Young Medical Scientists; and a Grant-in-Aid from the Keio Medical Association. We would like to thank Miyuki Saito for technical assistance and Misako Horikawa and Ryoko Suzuki for preparation of the manuscript.

1 Mestas J, Hughes CC. Of mice and not men: differences between mouse and human immunology. J Immunol 2004; 172: 2731-2738.

2 Ito M, Hiramatsu H, Kobayashi K, Suzue K, Kawahata M, Hioki K et al. NOD/SCID/gamma(c)(null) mouse: an excellent recipient mouse model for engraftment of human cells. Blood 2002; 100: 3175-3182.

3 Shultz LD, Lyons BL, Burzenski LM, Gott B, Chen X, Chaleff S et al. Human Iymphoid and myeloid cell development in NOD/LtSz-scid IL2R gamma null mice engrafted with mobilized human hemopoietic stem cells. J Immunol 2005; 174: 6477-6489.

4 Ito R, Takahashi T, Katano I, Ito M. Current advances in humanized mouse models. Cell Mol Immunol 2012; 9: 208-214.

5 Ishikawa F, Yasukawa M, Lyons B, Yoshida S, Miyamoto T, Yoshimoto $\mathrm{G}$ et al. Development of functional human blood and immune systems in NOD/SCID/IL2 receptor \{gamma\} chain(null) mice. Blood 2005; 106: 1565-1573.

6 Matsumura T, Kametani Y, Ando K, Hirano Y, Katano I, Ito R et al. Functional CD5+ B cells develop predominantly in the spleen of NOD/ SCID/gammac(null) (NOG) mice transplanted either with human umbilical cord blood, bone marrow, or mobilized peripheral blood CD34+ cells. Exp Hematol 2003; 31: 789-797.

7 Hiramatsu H, Nishikomori R, Heike T, Ito M, Kobayashi K, Katamura K et al. Complete reconstitution of human lymphocytes from cord blood CD34+ cells using the NOD/SCID/gammacnull mice model. Blood 2003; 102: 873-880.

8 Shultz LD, Saito Y, Najima Y, Tanaka S, Ochi T, Tomizawa M et al. Generation of functional human T-cell subsets with HLA-restricted immune responses in HLA class I expressing NOD/SCID/IL2r gamma (null) humanized mice. Proc Natl Acad Sci USA 2010; 107: 13022-13027

9 Danner R, Chaudhari SN, Rosenberger J, Surls J, Richie TL, Brumeanu TD et al. Expression of HLA class II molecules in humanized NOD.Rag1KO.IL2RgcKO mice is critical for development and function of human T and B cells. PLoS ONE 2011; 6: e19826.

10 Shultz LD, Brehm MA, Garcia-Martinez JV, Greiner DL. Humanized mice for immune system investigation: progress, promise and challenges. Nat Rev Immunol 2012; 12: 786-798.

11 Ito R, Katano I, Kawai K, Hirata H, Ogura T, Kamisako T et al. Highly sensitive model for xenogenic GVHD using severe immunodeficient NOG mice. Transplantation 2009; 87: 1654-1658.

12 Pino S, Brehm MA, Covassin-Barberis L, King M, Gott B, Chase TH et al. Development of novel major histocompatibility complex class I and class II-deficient NOD-SCID IL2R gamma chain knockout mice for modeling human xenogeneic graft-versus-host disease. Methods $\mathrm{Mol}$ Biol 2010; 602: 105-117.

13 Inozume T, Hanada K, Wang QJ, Ahmadzadeh M, Wunderlich JR, Rosenberg SA et al. Selection of CD8+PD-1+ lymphocytes in fresh human melanomas enriches for tumor-reactive T cells. J Immunother 2010; 33: 956-964.

14 Yaguchi T, Goto Y, Kido K, Mochimaru H, Sakurai T, Tsukamoto N et al. Immune suppression and resistance mediated by constitutive 
activation of Wnt/beta-catenin signaling in human melanoma cells. J Immunol 2012; 189: 2110-2117.

15 Johnson LA, Morgan RA, Dudley ME, Cassard L, Yang JC, Hughes MS et al. Gene therapy with human and mouse T-cell receptors mediates cancer regression and targets normal tissues expressing cognate antigen. Blood 2009; 114: 535-546.

16 Kawakami Y, Eliyahu S, Delgado CH, Robbins PF, Rivoltini L, Topalian SL et al. Cloning of the gene coding for a shared human melanoma antigen recognized by autologous $T$ cells infiltrating into tumor. Proc Natl Acad Sci USA 1994; 91: 3515-3519.

17 Inozume T, Yaguchi T, Furuta J, Harada K, Kawakami Y, Shimada S. Melanoma cells control anti-melanoma CTL responses via interaction between TIGIT and CD155 in the effector phase. J Invest Dermatol 2016; 136: 255-263.

18 Furuta J, Inozume T, Harada K, Shimada S. CD271 on melanoma cell is an IFN-gamma-inducible immunosuppressive factor that mediates downregulation of melanoma antigens. J Invest Dermatol 2014; 134: 1369-1377.

19 King MA, Covassin L, Brehm MA, Racki W, Pearson T, Leif J et al. Human peripheral blood leucocyte non-obese diabetic-severe combined immunodeficiency interleukin-2 receptor gamma chain gene mouse model of xenogeneic graft-versus-host-like disease and the role of host major histocompatibility complex. Clin Exp Immunol 2009; 157: 104-118.

20 Okada M, Okuno Y, Hashimoto S, Kita Y, Kanamaru N, Nishida Y et al. Development of vaccines and passive immunotherapy against SARS corona virus using SCID-PBL/hu mouse models. Vaccine 2007; 25: 3038-3040.

21 Yahata T, Ando K, Nakamura Y, Ueyama Y, Shimamura K, Tamaoki N et al. Functional human $\mathrm{T}$ lymphocyte development from cord blood CD34+ cells in nonobese diabetic/Shi-scid, IL-2 receptor gamma null mice. J Immunol 2002; 169: 204-209.

22 Traggiai E, Chicha L, Mazzucchelli L, Bronz L, Piffaretti JC, Lanzavecchia A et al. Development of a human adaptive immune system in cord blood cell-transplanted mice. Science 2004; 304: 104-107.

23 Jaiswal S, Pearson T, Friberg H, Shultz LD, Greiner DL, Rothman AL et al. Dengue virus infection and virus-specific HLA-A2 restricted immune responses in humanized NOD-scid IL2rgammanull mice. PLoS One 2009; 4: e7251.

24 Serra-Hassoun $M$, Bourgine $M$, Boniotto $M$, Berges J, Langa $F$, Michel ML et al. Human hematopoietic reconstitution and HLArestricted responses in nonpermissive alymphoid mice. J Immunol 2014; 193: 1504-1511.

25 Billerbeck E, Horwitz JA, Labitt RN, Donovan BM, Vega K, Budell WC et al. Characterization of human antiviral adaptive immune responses during hepatotropic virus infection in HLA-transgenic human immune system mice. J Immunol 2013; 191: 1753-1764.

26 Strowig T, Gurer C, Ploss A, Liu YF, Arrey F, Sashihara J et al. Priming of protective $T$ cell responses against virus-induced tumors in mice with human immune system components. J Exp Med 2009; 206: 1423-1434.

$27 \mathrm{Yu}$ Cl, Gallegos M, Marches F, Zurawski G, Ramilo O, Garcia-Sastre A et al. Broad influenza-specific CD8+ T-cell responses in humanized mice vaccinated with influenza virus vaccines. Blood 2008; 112 : 3671-3678.

28 Spranger S, Frankenberger B, Schendel DJ. NOD/scid IL-2Rg(null) mice: a preclinical model system to evaluate human dendritic cellbased vaccine strategies in vivo. J Trans/ Med 2012; 10: 30.

$29 \mathrm{Gao} \mathrm{H,} \mathrm{Li} \mathrm{K,} \mathrm{Tu} \mathrm{H,} \mathrm{Pan} \mathrm{X,} \mathrm{Jiang} \mathrm{H,} \mathrm{Shi} \mathrm{B} \mathrm{et} \mathrm{al.} \mathrm{Development} \mathrm{of} \mathrm{T} \mathrm{cells}$ redirected to glypican-3 for the treatment of hepatocellular carcinoma. Clin Cancer Res 2014; 20: 6418-6428.

30 Shirakura Y, Mizuno Y, Wang L, Imai N, Amaike C, Sato E et al. T-cell receptor gene therapy targeting melanoma-associated antigen-A4 inhibits human tumor growth in non-obese diabetic/SCID/gammacnull mice. Cancer Sci 2012; 103: 17-25.

31 Ashizawa T, lizuka A, Nonomura C, Kondou R, Maeda C, Miyata H et al. Antitumor effect of programmed death-1 (PD-1) blockade in humanized the NOG-MHC double knockout mouse. Clin Cancer Res 2017; 23: 149-158.

32 Katano I, Takahashi T, Ito R, Kamisako T, Mizusawa T, Ka Y et al. Predominant development of mature and functional human NK cells in a novel human IL-2-producing transgenic NOG mouse. J Immunol 2015; 194: 3513-3525.

Supplementary Information for this article can be found on the Cellular \& Molecular Immunology website (http://www.nature.com/cmi) 